\title{
A comprehensive method to elucidate pyoverdines produced by fluorescent Pseudomonas spp. by UHPLC-HR-MS/MS
}

\author{
${\text { Karoline } \text { Rehm }^{1} \text { (D) Vera Vollenweider }}^{2}$ (D) $\cdot$ Rolf Kümmerli $^{2}$ (D) $\cdot$ Laurent Bigler $^{1}$ (D)
}

Received: 1 December 2021 / Revised: 9 January 2022 / Accepted: 15 January 2022 / Published online: 27 January 2022

(c) The Author(s) 2022

\begin{abstract}
Microbial secondary metabolites represent a rich source for drug discovery, plant protective agents, and biotechnologically relevant compounds. Among them are siderophores, iron-chelating molecules, that show a great influence on bacterial community assembly and the potential to control pathogen invasions. One of such a siderophore is pyoverdine that is produced by fluorescent Pseudomonas members and consists of different peptide chains specific to each bacterial species. The identification and structural elucidation of such suites of siderophores remain widely underexplored as general high-throughput analytical protocols are missing. Therefore, a dedicated method was established allowing a rapid localization and structural elucidation of pyoverdines. Liquid bacterial culture samples were purified by an easy small-scale solid-phase extraction (SPE). Ultra-high-performance liquid chromatography high-resolution tandem mass spectrometry (UHPLC-HR-MS/MS) separated highly polar pyoverdines and their derivatives. All ion fragmentation (AIF) generated mass spectra containing the characteristic fragments of the biological precursor of pyoverdine, ferribactin. This led to the revelation of the mass of secreted pyoverdines. Targeted MS/MS experiments at multiple collision energies accomplished the full structure elucidation of the pyoverdine peptide chain. A mass calculator and a fragmentation predictor facilitated greatly the interpretation of MS/ MS spectra by providing accurate masses for a straightforward comparison of measured and theoretical values. The method was successfully validated using four well-known pyoverdines with various peptide chains. Finally, the applicability was proven by the analysis of 13 unknown pyoverdines secreted by sampled bacterial cultures. Among these, 4 novel pyoverdine peptide chains were discovered and are herein reported for the first time.
\end{abstract}

Keywords Siderophore $\cdot$ Pyoverdine $\cdot$ Ferribactin $\cdot$ Isopyoverdine $\cdot$ Pseudomonas $\cdot$ UHPLC-MS $\cdot$ High-throughput structure elucidation pipeline

\section{Introduction}

Microorganisms produce a vast number of secondary metabolites that play key roles in microbial physiological processes, and for growth and survival. Secondary metabolites are low-molecular-weight compounds with various bioactive properties specific to the species that exudes them. Among them are toxins to control competing organisms, compounds for metal transport, quorum-sensing molecules for chemical

Laurent Bigler

laurent.bigler@chem.uzh.ch

1 Department of Chemistry, University of Zurich, Winterthurerstr. 190, 8057 Zurich, Switzerland

2 Department of Quantitative Biomedicine, University of Zurich, Winterthurerstr. 190, 8057 Zurich, Switzerland communication, biosurfactants to modulate group motility, and many more [1-5].

Over the last years, the interest for microbial secondary metabolites increased immensely. They serve as a primary source for drug discovery resulting in novel antibiotics, antitumor drugs, or immunosuppressants [6, 7]. In the field of biotechnical engineering, biosurfactants gained popularity as a sustainable and environmentally friendly alternative to synthetic surfactants [8]. Last but not least, iron-chelating metabolites are utilized for medicinal and agricultural applications [9]. These iron carriers are called siderophores, possess a high iron-binding affinity $\left(>10^{30} \mathrm{M}^{-1}\right)$, and are with over 500 structures chemically diverse [10]. Recent studies revealed the great potential of siderophores as probiotics to protect plants from pathogen infections [11] and to protect bacterial communities from pathogen invasion [12]. Despite the clear efficiency of certain siderophores 
to inhibit pathogen growth, the chemical structure of these siderophores remains mostly unknown.

To facilitate research on bacterial secondary metabolites, we present a novel method based on UHPLC-MS/MS for the high-throughput characterization of pyoverdines from crude bacterial culture extracts. Pyoverdines are iron-scavenging chromopeptides and are a siderophore solely produced by fluorescent Pseudomonas species [13]. Each fluorescent Pseudomonas species produces its own type of pyoverdine varying in length and amino acid sequence. This is why over 60 pyoverdines were discovered until today and many unknown pyoverdine types are yet to be characterized [14]. They are a major force that can promote or impede microbial invasions in bacterial communities. However, the chemical structures of promoting or inhibiting pyoverdines were not elucidated in the respective studies $[11,12]$ as their characterization is demanding and time consuming.

Nuclear magnetic resonance (NMR) spectroscopy and tandem mass spectrometry (MS/MS) deliver the highest level of confidence for a full characterization of a secreted pyoverdine. However, bacterial liquid cultures are complex mixtures requiring a tedious purification of their components before analysis. Furthermore, a bacterium is not only producing a single pyoverdine structure but also multiple derivatives and related compounds complicating the matter.

Taking a closer look at the chemical structure of these compounds (Fig. 1), pyoverdines and their derivatives can be defined as chromopeptides that can be separated into three parts: a sidechain $\mathrm{R}_{\mathrm{SC}}$ (Fig. 1a), a chromophore core (Fig. 1b), and a peptide part Pep (Fig. 1c) [15]. In bacterial
Fig. 1 Building blocks of pyoverdines and their derivatives. a Varieties of acyl side chains found in bacterial extracts. Note: Position of the hydroxy group of 3/4 (highlighted in blue) is not resolved and could be either one of the two positions [17]. b Varieties of chromophores encountered in natural occurring pyoverdine derivatives [16]. c Structurally versatile peptide chain varied by bacterial strain

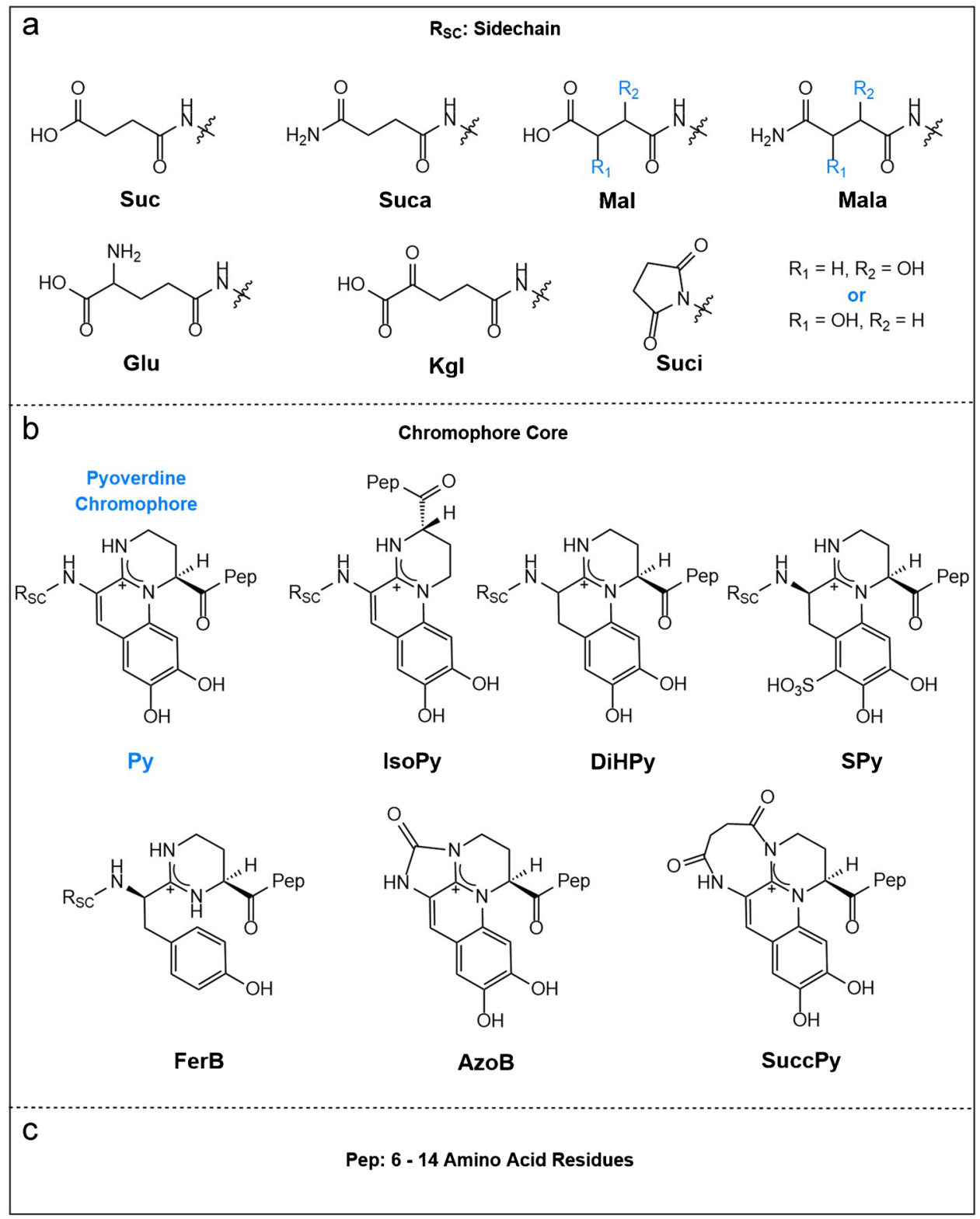


extracts, variations of the structure in all three parts have been found. For the side chain, not only succinic acid (Suc) was identified but also succinic amide (Suca), malic acid/ amide (Mal, Mala), $\alpha$-ketoglutaric acid (Kgl), glutamic acid (Glu), and a succinic imide derivative (Suci). Overall, succinic acid and succinic amide are the most frequently encountered side chains [15]. Pyoverdine itself is only characterized by a 2,3-diamino-6,7-dihydroxyquinoline unit (Py) but certain biological precursors and other modifications are also commonly detected. Among them are isopyoverdine (IsoPy), dihydro-pyoverdine (DiHPy), dihyodro-pyoverdine-7-sulfonic acid (SPy), ferribactin (FerB), azotobactin (AzoB), and succinopyoverdine (SuccPy) [16]. Finally, a linear or cyclic peptide chain consisting of 6-14 amino acid residues is linked to the core. While the sidechain and the chromophore units are mostly conserved, the sequence of the peptide chain varies greatly depending on the bacterial species.

The most popular and established approach to determine the structure of pyoverdines is based on electrospray ionization (ESI) in combination with collision-induced dissociation of selected ions $[15,18,19]$. For the chromophore core, characteristic fragments are used for its identification. Apart from that, mostly B and $\mathrm{Y}^{\prime \prime}$ fragment ions are detected that allow the elucidation of the peptide chain [15].

This structure elucidation is a complex task as pyoverdine and its derivatives are often detected as a mixture containing various side chains, chromophores and in some cases both linear and cyclized entities. Purification of pyoverdines has been mostly conducted by large-scale solid-phase purification on a XAD-4 resin [20] or by semi-preparative HPLC [21] consuming time and resources. The structure of the compounds of interest was then investigated with MS/MS by continuous flow injection without prior LC separation [22-24]. In the LC-MS/MS studies to date, crude extracts were analyzed, whereby only a few specific pyoverdines have been investigated $[18,19]$. Overall, the need of further method optimization and an easy protocol for fragmentation interpretation is required to ensure the accessibility of highthroughput structure elucidation.

In this work, a generalized high-throughput UHPLCMS/MS pipeline was developed to characterize pyoverdines and their derivatives in bacterial liquid cultures. Four well-known pyoverdines differing in their peptide chain and $\mathrm{C}$-terminal end were used to optimize purification, $\mathrm{LC}$, and MS conditions and to validate the procedure. Minimal sample amounts of bacterial cultures $(500 \mu \mathrm{L})$ were extracted by solid-phase extraction (SPE) and were directly submitted to liquid chromatography. The polar components of the bacterial extracts were separated with a high peak capacity and analyzed with MS/MS in only 15 min per sample. The high mass accuracy of a Q Exactive MS instrument, pyoverdine fragmentation at multiple collision energies, and a fully exploited duty cycle ensured a high sensitivity and simplified annotation of B and $\mathrm{Y}^{\prime \prime}$ ions. For a rapid MS/MS interpretation, a new pyoverdine fragmentation predictor programmed in Excel was created allowing a quick comparison between measured and theoretical masses. Applicability of the method was proven by successfully analyzing the unknown pyoverdines found in the bacterial extract of 13 fluorescent Pseudomonas species.

\section{Materials and methods}

\section{Chemicals and reagents}

Commercial pyoverdines from P. fluorescens with reported structures (Py SA, CAS RN 8062-00-8, >90\%) and 2,2'-bipyridine ( $\geq 99 \%)$ were obtained from Sigma Aldrich (Buchs, SG, Switzerland). Acetonitrile $\left(\mathrm{CH}_{3} \mathrm{CN}\right)$ and methanol $(\mathrm{MeOH})$ were obtained from Biosolve (ULC grade, Valkenswaard, Netherlands) and formic acid from Fluka (LC-MS grade, Buchs, SG, Switzerland). Ultrapure water $\left(<2\right.$ ppb TOC) was produced using a Milli- ${ }^{\circledR}$ Advantage A10 water purification system (Merck, Bedford, MA, USA). Pierce LTQ Velos ESI Positive Ion Calibration Solution was purchased from Thermo Fisher (Waltham, MA, USA). For the growth of bacteria, a casamino acid solution (CAA) was used as a nutrient medium. This aqueous solution $(1 \mathrm{~L})$ was made from vitamin-free casein acid hydrolysate $(10.0 \mathrm{~g})$, $\mathrm{K}_{2} \mathrm{HPO}_{4} \cdot 3 \mathrm{H}_{2} \mathrm{O}(1.18 \mathrm{~g})$, and $\mathrm{MgSO}_{4} \cdot 7 \mathrm{H}_{2} \mathrm{O}(0.25 \mathrm{~g})$. The listed ingredients were bought from Sigma Aldrich (Buchs, SG, Switzerland).

\section{Bacterial cultures}

All cultures originated from the Rolf Kümmerli strain collection (University of Zurich) and were stored as glycerol stocks at $-80^{\circ}$. Among them were the $P$. aeruginosa strains PAO1, 1-60, and 206-12 producing pyoverdine types 1, 2, and 3 , respectively $[25,26]$. As the chemical structures of all three types have been characterized, the pyoverdines of these strains were used together with the commercially available pyoverdine (Py SA) to establish and verify our structure elucidation pipeline. Furthermore, 13 Pseudomonas environmental isolates sampled from soil and pond habitats on the campus of the University of Zurich Irchel $\left(47.40^{\circ} \mathrm{N}, 8.54^{\circ}\right.$ $\mathrm{E}$; Switzerland) were investigated. Their sampling procedure and isolation can be viewed in [27]. These environmental isolates are known to produce pyoverdine, but it is unknown which type they make. 


\section{Growth of liquid cultures}

Bacteria were transferred from the glycerol stock solution into a Falcon tube containing lysogeny broth $(8 \mathrm{~mL})$ using a sterile inoculation loop and were incubated overnight at $28{ }^{\circ} \mathrm{C}$ under vigorous shaking $(170 \mathrm{rpm})$. Next, the cultures were centrifuged (7500 rcf, $3 \mathrm{~min}$ ) and the supernatant was discarded. The remaining pellet was washed twice with $0.8 \%$ $\mathrm{NaCl}(8 \mathrm{~mL})$ by vortexing, subsequent centrifugation $(7500$ rcf, $3 \mathrm{~min}$ ), and removal of the wash solution. An aqueous $\mathrm{NaCl}$ solution $(0.8 \%)$ was added to the washed pellet until an optical density at $600 \mathrm{~nm}$ (OD600) of 1 absorbance unit (AU) was reached. $2 \mathrm{~mL}$ of the resuspended culture were added to $5 \%$ CAA $(500 \mathrm{~mL})$ supplemented with $250 \mu \mathrm{M}$ 2,2'-bipyridine $(1.25 \mathrm{~mL})$, an iron chelator that induces pyoverdine production in all Pseudomonas isolates, and grown at $28^{\circ} \mathrm{C}$ under vigorous shaking $(170 \mathrm{rpm})$. After $72-120 \mathrm{~h}$, the liquid culture was centrifuged (7500 rcf, $3 \mathrm{~min}$ ) and the pyoverdine containing supernatant was sterile-filtered through a $0.22 \mu \mathrm{m}$ filter before storage at $-20{ }^{\circ} \mathrm{C}$.

\section{Sample preparation}

All biological samples were extracted by SPE using Strata-X $(1 \mathrm{~mL}, 30 \mathrm{mg}$ ) polymeric reversed-phase cartridges (Phenomenex, Torrance, CA, USA). The cartridges were conditioned with $1 \mathrm{~mL} \mathrm{MeOH}$ followed by equilibration with $1 \mathrm{~mL} \mathrm{H}_{2} \mathrm{O}$. A solution of $5 \mu \mathrm{L}$ concentrated formic acid was added to the thawed supernatant samples $(500 \mu \mathrm{L})$ inside an Eppendorf vial and vortexed for $5 \mathrm{~s}$. The samples were then loaded onto the sorbent and subsequently washed with $\mathrm{H}_{2} \mathrm{O}$ $(2 \times 0.3 \mathrm{~mL})$. The pyoverdine fractions were finally eluted with $\mathrm{H}_{2} \mathrm{O} / \mathrm{MeOH} 7: 3+0.1 \% \mathrm{HCOOH}(2 \times 0.3 \mathrm{~mL})$. No further reconstitution was performed and the elute was either directly injected for measurement or stored at $-20{ }^{\circ} \mathrm{C}$ until use.

The pyoverdine reference material purchased from Sigma Aldrich (Py SA) was dissolved in $\mathrm{H}_{2} \mathrm{O} / \mathrm{MeOH} 1: 1$ at $50 \mu \mathrm{g} /$ $\mathrm{mL}$ concentration.

Between 5 and $20 \mu \mathrm{L}$ of the sample was injected for analysis.

\section{Chromatography}

Liquid-chromatography was performed on an UltiMate 3000 UHPLC (Thermo Fisher, Waltham, MA, USA) build from a binary RS pump, an XRS open autosampler, and a temperature-controllable RS column oven. Chromatographic separation was achieved at $25^{\circ} \mathrm{C}$ on an ACQUITY UPLC HSS C18 Column (100 ̊̊, $1.8 \mu \mathrm{m}, 2.1 \times 100$ mm, Waters, Milford, USA). Eluent A consisted of $\mathrm{H}_{2} \mathrm{O}+0.1 \% \mathrm{HCOOH}$ and $\mathrm{B}$ of $\mathrm{CH}_{3} \mathrm{CN}+0.1 \% \mathrm{HCOOH}$. The following gradient was applied at a constant flowrate of $0.4 \mathrm{~mL}$ : (i) $4 \% \mathrm{~B}$ isocratic from $0.0-0.5 \mathrm{~min}$; (ii) linear increase to $11 \% \mathrm{~B}$ until $5.25 \mathrm{~min}$; (iii) linear increase to $95 \%$ until $7.0 \mathrm{~min}$; (iv) holding $95 \%$ B until 10.0 min; (vi) back to the starting conditions of $4 \% \mathrm{~B}$ until $10.5 \mathrm{~min}$; and (vii) equilibration for $4.5 \mathrm{~min}$ until the next run.

\section{Mass spectrometry}

The UHPLC was coupled to a Q Exactive hybrid quadrupole-Orbitrap mass spectrometer (Thermo Fisher Scientific, Waltham, MA, USA) equipped with a heated ESI source (position B). The (+)-ESI parameters were as followed: spray voltage $3.5 \mathrm{kV}$, sheath gas $50 \mathrm{~L} / \mathrm{min}$, auxiliary gas $13 \mathrm{~L} / \mathrm{min}$, sweep gas $3 \mathrm{~L} / \mathrm{min}\left(\mathrm{N}_{2}\right)$, capillary temperature $260{ }^{\circ} \mathrm{C}$, auxiliary gas temperature $450{ }^{\circ} \mathrm{C}, \mathrm{S}$-lens RF level 55.0. Samples were measured under two different conditions: (1) Alternative full scan (FS)/all ion fragmentation (AIF, higher-energy collisional dissociation of all ionized molecules without prior mass filtering), 140,000 full width half maximum resolution (FWHM at $\mathrm{m} / \mathrm{z}, 200$ ), IT $_{\max }$ $500 \mathrm{~ms}$, AGC target $3 \mathrm{e} 6$ for FS as well as AIF, mass ranges from $m / z, 140-2000$ (FS) and $m / z$ 133.3-1900 (AIF), and normalized collision energy (NCE) of 50. (2) Alternative FS/parallel reaction monitoring (PRM), resolution of 70,000 FWHM with IT $_{\max } 200 \mathrm{~ms}$ (FS) and 35,000 FWHM with IT $_{\text {max }} 110$ ms PRM (PRM), AGC target of $3 \mathrm{e} 6$ (FS) and 5e5 (PRM), non-stepped NCE at 20, 25, 30, 35, 50, and 100, loop count 12 . The targeted ions that were used for fragmentation and elucidation of the pyoverdine peptide chains of the bacterial extracts analyzed in this work can be found in the supplementary Table S1.

Using a Pierce LTQ ESI positive ion calibration solution (Thermo Fisher Scientific, Waltham, MA, USA) for external calibration, a mass deviation below 5 ppm was tolerated. Xcalibur 4.1 was used for data acquisition and interpretation. An Excel calculator was used to predict the $m / z$ values of masses and fragments of pyoverdines and related compounds (available in the supplementary).

\section{Results and discussion}

\section{Pyoverdine purification by SPE}

To avoid clogging injectors and column frits through proteins and to minimize matrix effects [28], a sample preparation protocol based on SPE was developed. Different SPE sorbents were evaluated: the Strata-X, Strata-X-C, and Strata-X-CW. Thereby, the Strata-X delivered the highest analyte response. Afterwards, the solvent composition for the washing and elution step was adjusted testing various percentages of $\mathrm{MeOH}$ in water. No analyte was lost 
during the washing step with pure $\mathrm{H}_{2} \mathrm{O}$. Using $30 \% \mathrm{MeOH}$ in $0.1 \%$ formic acid acidified $\mathrm{H}_{2} \mathrm{O}$ for the pyoverdine elution, a recovery of approximately $80 \%$ was obtained. It was refrained to further increase the elution strength as interferents from the liquid culture medium were observed that would impact the high robustness of the method.

Using the optimized method, pyoverdines of all bacterial supernatants were purified successfully. Compared to the traditional Amberlite XAD-4 resin purification [15], lower volumes of supernatant $(0.5 \mathrm{~mL}$ vs. $500 \mathrm{~mL})$, total solvent $(3.2 \mathrm{~mL}$ vs. $>200 \mathrm{~mL})$, and a shorter time ( $15 \mathrm{~min}$ vs. $>1 \mathrm{~h})$ are necessary per sample. Even more time can be saved by extracting up to 12 supernatants in parallel with one SPE manifold making small-scale SPE purification suitable for high-throughput analysis. Compared to the single available SPE method for pyoverdines [18], only a single washing step and no reconstitution is required.

\section{Chromatographic separation of pyoverdine extracts}

After testing various column materials as well as eluent additives, the HSS C18 column using $0.1 \%$ formic acid as a modifier and $\mathrm{CH}_{3} \mathrm{CN}$ as the organic phase gave the best separation for all extracted samples. All pyoverdines and their derivatives elute in less than $6 \mathrm{~min}$. An exemplary chromatogram of the sample S3e20 is illustrated in Fig. 2a showing the complexity of a crude bacterial extract. Peak shapes are highly symmetrical and sharp as seen in the extracted ion chromatograms of the Glu-FerB, Suc-Py, and Suca-Py compounds found in sample S3e20 (Fig. 2b). In general, peak width ranged from 0.1 to $0.2 \mathrm{~min}$ depending on the analyte. Slight peak tailing was observed in few cases like for Glu-FerB in Fig. 2b.

A full list of retention times of all detected Glu-FerB, Suc-Py, and Suca-Py compounds in each bacterial sample is given in the electronic supplementary (Table S2). The identification of the relevant peaks is explained in the next sections. Each compound has a very specific retention time covering a broad range from 1.33 to $5.78 \mathrm{~min}$. All peptides were sufficiently retained on the column material despite their high polarity. A full baseline separation of Suc-Py and its corresponding Suca-Py (with an identical peptide chain) was achieved in all cases (where both analytes were present). Comparing the retention times of all Suca-Py compounds to their succinic acid side chain derivatives, the Suca-Py compounds elute in average around $0.67 \pm 0.06$ min earlier. Therefore, the retention time of one out of two compounds can be easily estimated from the retention time of the other. In each extract, Glu-FerB were fully or partially separated from their corresponding Suca-Py. Furthermore, Glu-IsoPy and Glu-FerB with the same side chain were also separated in the single bacterial strain $(3 \mathrm{C} 16)$ that produced both compounds. Therefore, a good selectivity of the column material based on the chromophore core is given, too.

Compared to already published methods, we present not only a chromatographic method with a shorter run time but also demonstrate a higher separation power. For example, Wei and Aristilde [19] use a 30 min gradient with a shorter $50 \mathrm{~mm}$ Hypersil GOLD column. Baune et al. [18], on the other hand, employ a $150 \mathrm{~mm}$ long iHILIC Fusion column taking $40 \mathrm{~min}$ for a single run. In both studies, only few selected pyoverdines have been analyzed. Here, we have demonstrated that our separation method is suitable for a general application by analyzing a large number of pyoverdines with various structures and polarities.

\section{Identifying pyoverdines and their derivatives in complex samples}

Bacterial supernatants are a complex mixture even after a SPE purification (see Fig. 2). A common approach to localize pyoverdines in a HPLC chromatogram is by application of all ion fragmentation (AIF) to generate pyoverdine
Fig. 2 a Base peak chromatogram of a SPE purified bacterial extract labeled with the ID S3e20. b Normalized extracted ion chromatograms of Glu-FerB $(\mathrm{m} / \mathrm{z}$ 656.31295), Suca-Py $(\mathrm{m} / \mathrm{z}$ 647.28948), and Suc-Py $(\mathrm{m} / \mathrm{z}$ 647.78149 ) found in the sample $\mathrm{S} 3 \mathrm{e} 20$

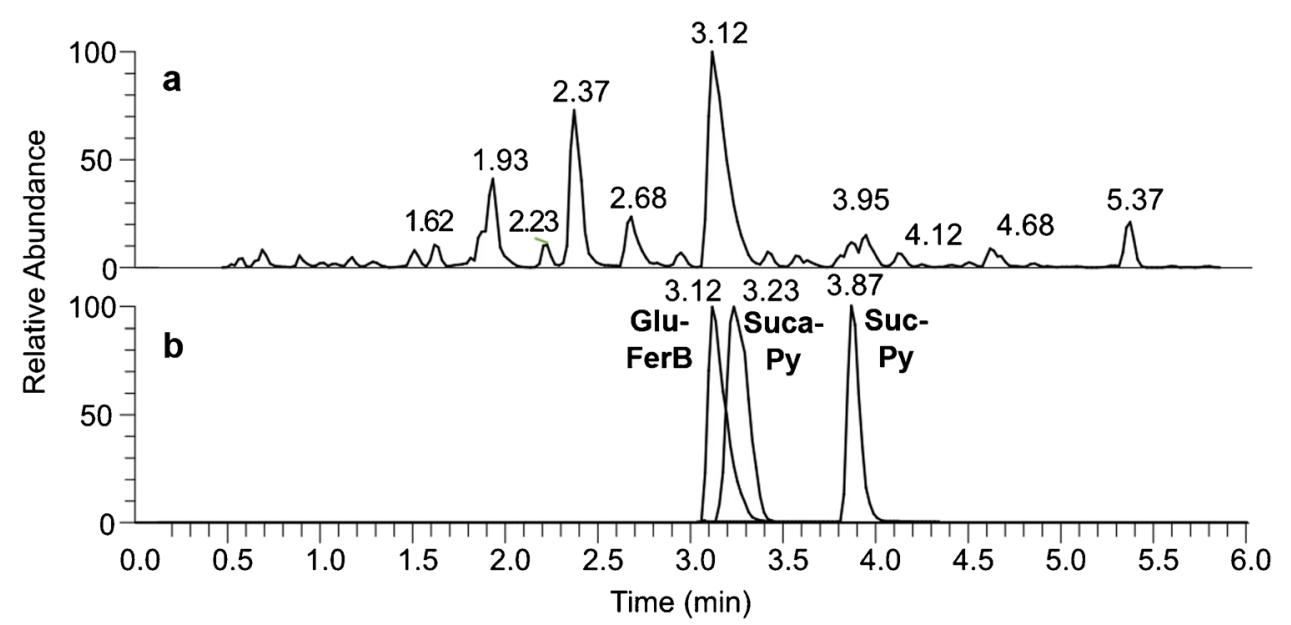


characteristic fragments and compare their retention time to possible precursor masses such as in the publication of Wei and Aristilde [19] or Budzikiewicz et al. [15]. This fragment is found at $m / z 204.0768\left(\mathrm{C}_{10} \mathrm{H}_{10} \mathrm{O}_{2} \mathrm{~N}_{3}\right)(\mathbf{a})$. It has its origin in a retro-Diels-Alder reaction of the pyoverdine chromophore, thus making it highly specific (Fig. 3) [16].

This approach is not straightforward for complex samples containing several types and/or a low quantity of pyoverdines. The problem is exemplified in Fig. 4: While two clear pyoverdine fragment signals are found in the extract of PAO1, up to nine peaks are detected for the extract of 3A06, which are all potential pyoverdine candidates. The fact that pyoverdines might exist with various side chains complicates the finding of a good starting point. Related derivatives such as azotobactin and succinopyoverdin produce also $\mathrm{m} / \mathrm{z} 204$ fragment ions even if in lower abundance [16]. Fragmenting each potential precursor mass and interpreting their MS/MS spectra would take several measurements and a lot of time.

Hence, a new procedure was implemented. Referring anew to the work of Budzikiewicz et al. [16], ferribactin, the biological precursor of pyoverdine, exists in almost all natural cases with a glutamic acid side chain (Glu-FerB). Ferribactins give rise to a tirade of characteristic fragments $(\mathrm{m} / \mathrm{z}$ $305.1452(\mathbf{b}), 170.0924(\mathbf{c})$, and $136.0757(\mathbf{d}))$ as shown in Fig. 5 (adapted from [16]).

In our study, the $m / z 136$ ions were the most abundant in all cases. Extracting this $\mathrm{m} / \mathrm{z}$ value, one dominant peak is visible as in the chromatogram of the PAO1 and the 3A06
Fig. 3 Fragmentation reaction of the pyoverdine chromophore resulting in the characteristic fragment $m / z 204\left(R_{\mathrm{SC}}=\right.$ side chain)

Fig. 4 Visual comparison of the base peak chromatograms of the UHPLC-MS analyzed bacterial SPE extracts of PAO1 (a) and 3A06 (b) together with the extracted ion chromatograms of the fragments characteristic for pyoverdine $(\mathrm{m} / \mathrm{z}, 204)$ and ferribactin $(\mathrm{m} / \mathrm{z}, 136)$ measured in the AIF experiment<smiles></smiles>

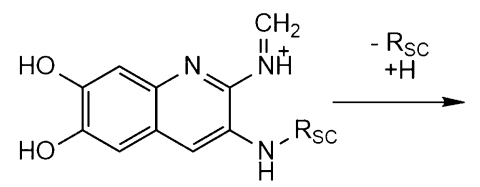<smiles>C=[N+]c1nc2cc(O)c(O)cc2cc1N</smiles>

a
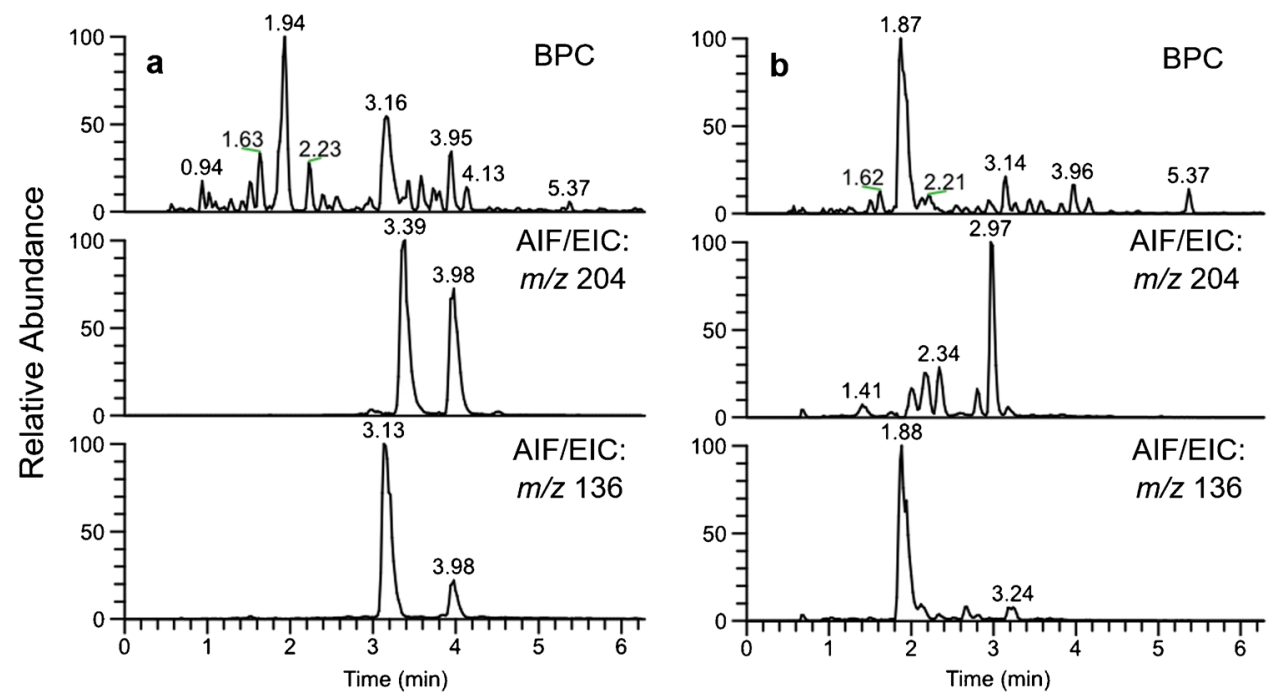<smiles>NC(=Cc1ccc(O)cc1)C1NCCC(C(=O)N=CCO)N1</smiles>

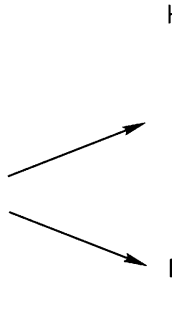

$\mathrm{HO}$

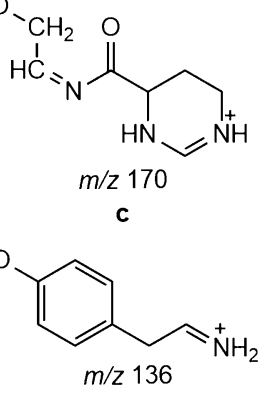

d 
extract (see Fig. 4). Knowing the ferribactin precursor mass, the mass for all hypothetical pyoverdines with various side chains can be derived easily. The theoretical mass difference of Glu-FerB to its corresponding Suc-Py and Suca-Py amounts -17.0629 and $-18.0470 \mathrm{Da}$, respectively. These pyoverdine masses can be extracted from the TIC. Afterwards, their identity can be confirmed by the pyoverdine characteristic fragment of the 2,3-diamino-6,7-dihydroxyquinoline chromophore at $m / z, 204.0768\left(\mathrm{C}_{10} \mathrm{H}_{10} \mathrm{O}_{2} \mathrm{~N}_{3}\right)$ in the AIF experiment. This pyoverdine identification approach worked for all samples and simplified the process of picking useful precursor masses for further fragmentation.

In two cases, an additional advantage of this procedure was found: For the supernatant of $3 \mathrm{C} 16$, clear fluorescence under UV light was visible but only a weak $\mathrm{m} / \mathrm{z}, 204$ fragment was detected. The bacterial strain was not discarded as a false assignment to the Pseudomonas species as ferribactin was detected. Instead, the possibility of the production of another pyoverdine derivative was considered and indeed, isopyoverdine was found. While only differing in the position of the peptide chain at the chromophore (Fig. 1, IsoPy), isopyoverdine decomposes to the fragment $\mathbf{e}$ with $m / z 230.0924\left(\mathrm{C}_{12} \mathrm{H}_{12} \mathrm{~N}_{3} \mathrm{O}_{2}{ }^{+}\right)$(Fig. 6) [16].

In Fig. 6, the fragmentation pattern in the lower mass range of the isopyoverdine with a glutamic acid side chain of sample $3 \mathrm{C} 16$ is compared to the fragmentation pattern of the succinic acid pyoverdine of S3c13. While the overall fragmentation behavior at $50 \mathrm{NCE}$ is similar below $\mathrm{m} / \mathrm{z}$
250 , the striking difference of an intense $m / z 230 \mathrm{vs} m / z 204$ signal is observable. Hence, the search for ferribactin fragment prevents the overlook of potential pyoverdine derivatives not owning highly abundant $m / z, 204$ fragments such as isopyoverdines or pyoverdines with an alpha-ketoglutaric acid side chain [16].

In a second case, another unexpected finding was encountered. While it is known that in seldom cases ferribactin might exist with a succinic amide side chain [16], a novel ferribactin without any sidechain was detected in sample S3a20 (H-FerB, see Fig. 7a). There, the most intense signal in the AIF belonged to ferribactin with no side chain (1) while the signal for the ferribactin with a glutamic acid side chain (2) was considerably lower in intensity (Fig. 7b). The identity of the side-chainless ferribactin was later proven by isolated MS/MS fragmentation (see supplementary Table S14) making it the first officially reported side-chainless ferribactin.

\section{Fragmentation behavior and choice of precursor ions}

For the MS/MS fragmentation of pyoverdines and its derivatives, parallel reaction monitoring was employed. Targeting only specific masses, the parent ion can be fragmented into several characteristic product ions inside the HCD cell. Thereby, a monoisotopic isolation is possible with an isolation window up to $m / z 0.4$.

Fig. 6 Fragmentation patterns in the lower regions of Suc-Py of $\mathrm{S} 3 \mathrm{c} 13\left([M+2 \mathrm{H}]^{2+}, 539.21017\right)$ (a) and Glu-IsoPy of $3 \mathrm{C} 16$ $\left([M+2 \mathrm{H}]^{2+}, 525.70472\right)(\mathbf{b})$ at an NCE of 50 highlighting the characteristic fragment of the chromophore unit

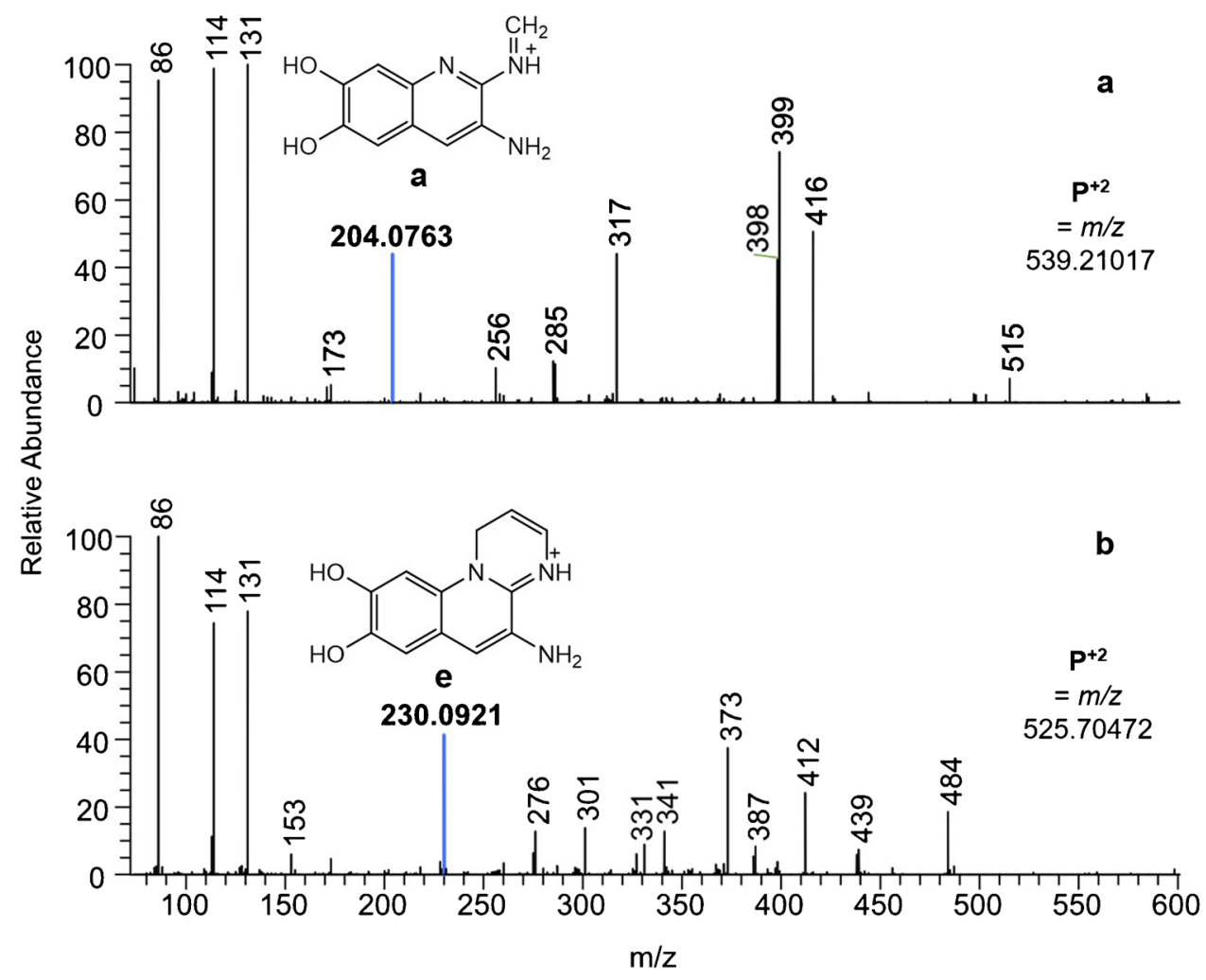


Fig. 7 a Chemical structure of Glu-FerB (1) and H-FerB (2) found in the sample S3a20. b Comparison of the base peak chromatogram of the full scan and the extracted ion chromatogram of the $m / z, 136$ fragment ions for sample S3a20<smiles>[R6]C=[R5]C=[R5]</smiles>

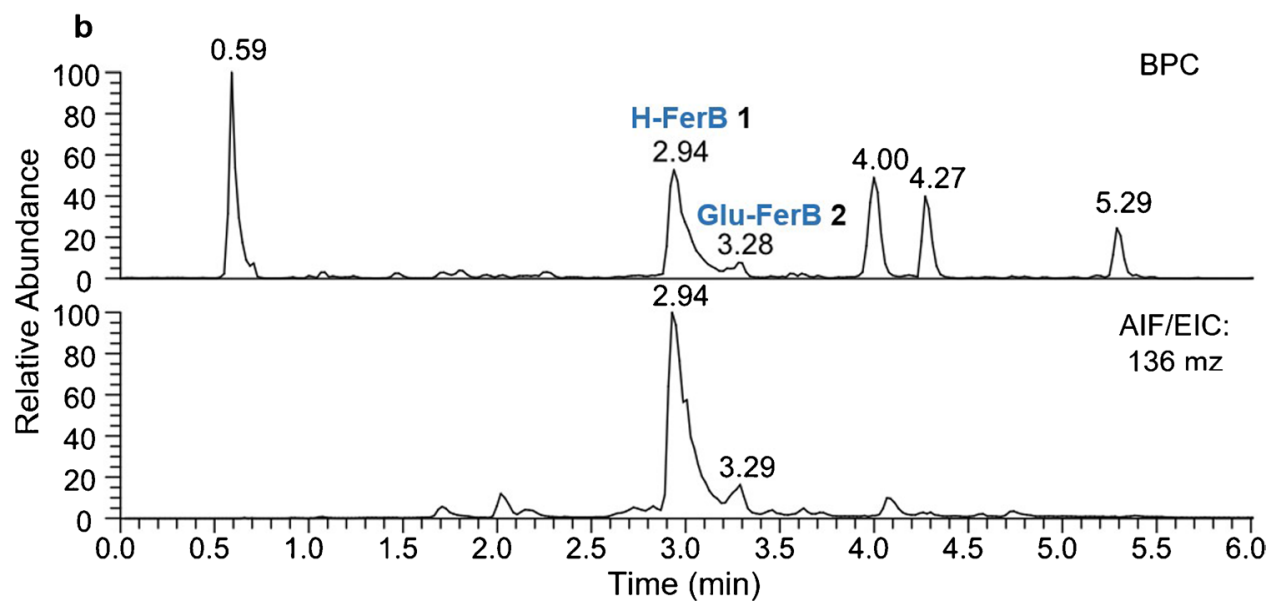

Table 1 Pyoverdines defined by their peptide chain analyzed in this study. Sum formulas and masses are based on the Suc-Py derivatives according to the recommendation of Meyer et al. [15] (Unusual amino acids: OHAsp, threo- $\beta$-hydroxy-Asp; OHOrn, $\mathrm{N}^{4}$-hydroxy-Orn
Ac(Fo)OHOrn, $\mathrm{N}^{4}$-acetyl-(formyl) OHOrn; cOHOrn, cyclo-OHOrn (3-amino-1-hydroxy-piperidone-2); aThr, allo-Thr) (* presence of a Thr instead of Thr reported in literature [14])

\begin{tabular}{|c|c|c|c|c|c|}
\hline Sample ID & Peptide chain & Sum formula Suc-Py & $\begin{array}{l}\text { Nom. mass } \\
\text { Suc-Py (Da) }\end{array}$ & {$[M+\mathrm{H}]^{+}$Suc-Py $(m / z)$} & $\begin{array}{l}{[M+2 \mathrm{H}]^{2+}} \\
\text { Suc-Py }(m / z)\end{array}$ \\
\hline Py SA & Ser-Lys-Gly-FoOHOrn-(Lys-FoOHOrn-Ser) & $\mathrm{C}_{49} \mathrm{H}_{72} \mathrm{~N}_{14} \mathrm{O}_{19}$ & 1160 & 1161.51764 & 581.26273 \\
\hline PAO1 & Ser-Arg-Ser-FoOHOrn-(Lys-FoOHOrn-Thr-Thr) & $\mathrm{C}_{55} \mathrm{H}_{83} \mathrm{~N}_{17} \mathrm{O}_{22}$ & 1333 & 1334.59768 & 667.80275 \\
\hline $1-60$ & Ser-FoOHOrn-Orn-Gly-aThr*-Ser-cOHOrn & $\mathrm{C}_{45} \mathrm{H}_{65} \mathrm{~N}_{13} \mathrm{O}_{19}$ & 1091 & 1092.45979 & 546.73381 \\
\hline $206-12$ & (Ser-Dab)-FoOHOrn-Gln-Gln-FoOHOrn-Gly & $\mathrm{C}_{48} \mathrm{H}_{67} \mathrm{~N}_{15} \mathrm{O}_{20}$ & 1173 & 1174.47650 & 587.74216 \\
\hline $3 \mathrm{~A} 06$ & Asp-e-Lys-OHAsp-Ser-Thr-Ser-Lys-cOHOrn & $\mathrm{C}_{52} \mathrm{H}_{76} \mathrm{~N}_{14} \mathrm{O}_{23}$ & 1264 & 1265.52860 & 633.26821 \\
\hline 3B19 & Asp-FoOHOrn-Lys-(Thr-Ala-Ala-Lys-FoOHOrn-Lys) & $\mathrm{C}_{61} \mathrm{H}_{93} \mathrm{~N}_{17} \mathrm{O}_{22}$ & 1415 & 1416.67593 & 708.84188 \\
\hline $3 \mathrm{C} 16$ & Asp-Ala-Asp-AcOHOrn-Ser-cOHOrn & $\mathrm{C}_{44} \mathrm{H}_{60} \mathrm{~N}_{11} \mathrm{O}_{20}$ & 1047 & 1048.38596 & 524.69689 \\
\hline 3D19 & Ala-AcOHOrn-Gly-Gly-Ser-Ala-OHAsp-Thr & $\mathrm{C}_{43} \mathrm{H}_{57} \mathrm{~N}_{12} \mathrm{O}_{22}$ & 1122 & 1123.41799 & 562.21291 \\
\hline $3 \mathrm{~F} 12$ & Lys-AcOHOrn-Gly-Thr-Thr-Gln-Gly-Ser-cOHOrn & $\mathrm{C}_{55} \mathrm{H}_{82} \mathrm{~N}_{16} \mathrm{O}_{22}$ & 1318 & 1319.58678 & 660.29730 \\
\hline $3 \mathrm{G} 07$ & Asp-FoOHOrn-Lys-(Thr-Ala-Ala-FoOHOrn-Lys) & $\mathrm{C}_{55} \mathrm{H}_{81} \mathrm{~N}_{15} \mathrm{O}_{21}$ & 1287 & 1288.58097 & 644.79440 \\
\hline S3a05 & Lys-AcOHOrn-Ala-Gly-aThr*-Ser-cOHOrn & $\mathrm{C}_{47} \mathrm{H}_{69} \mathrm{~N}_{13} \mathrm{O}_{18}$ & 1103 & 1104.49618 & 552.75200 \\
\hline $\mathrm{S} 3 \mathrm{a} 20$ & $\varepsilon$-Lys-OHAsp-Ala-aThr*-Ala-cOHOrn & $\mathrm{C}_{42} \mathrm{H}_{59} \mathrm{~N}_{11} \mathrm{O}_{17}$ & 989 & 990.41686 & 495.71234 \\
\hline S3b09 & Asp- $\varepsilon$-Lys-OHAsp-Ser-aThr*-Ala-Thr-Lys-cOHOrn & $\mathrm{C}_{56} \mathrm{H}_{83} \mathrm{~N}_{15} \mathrm{O}_{24}$ & 1349 & 1350.58136 & 675.79459 \\
\hline S3b16 & Ala-Lys-Thr-Ser-AcOHOrn-cOHOrn & $\mathrm{C}_{45} \mathrm{H}_{66} \mathrm{~N}_{12} \mathrm{O}_{17}$ & 1046 & 1047.47471 & 524.24127 \\
\hline S3c13 & Ser-Val-OHAsp-Gly-Thr-Ser-cOHOrn & $\mathrm{C}_{43} \mathrm{H}_{59} \mathrm{~N}_{11} \mathrm{O}_{20}$ & 1049 & 1050.40161 & 525.70472 \\
\hline $\mathrm{S} 3 \mathrm{e} 20$ & Ser-Lys-Ala-Ser-Ser-AcOHOrn-Ser-Ser-cOHOrn & $\mathrm{C}_{53} \mathrm{H}_{79} \mathrm{~N}_{15} \mathrm{O}_{23}$ & 1293 & 1294.55515 & 647.78149 \\
\hline S3g01 & Ala-AcOHOrn-Ala-Gly-Ser-Ala-OHAsp-Thr & $\mathrm{C}_{46} \mathrm{H}_{64} \mathrm{~N}_{12} \mathrm{O}_{22}$ & 1136 & 1137.43364 & 569.22073 \\
\hline
\end{tabular}


The pyoverdines from Py SA as well as from the SPE purified supernatants of PAO1, 1-60, and 206-12 cultures were used as reference and control compounds as their chemical structures were known in advance (see Table 1). Both cyclic and linear pyoverdines with different peptide chains were hence available to optimize a broad band of collision energies that generates interpretable fragment spectra for all pyoverdine types. The $[M+2 \mathrm{H}]^{2+}$ ions of SucPy from Py SA, PAO1, 1-60, and 206-12 were fragmented stepwise from 20-100 NCE to determine the optimal normalized collision energy (NCE). The best collision energies turned out to be 20,25,30, and 35 (a.u.). Additionally, fragmentation spectra were recorded at an NCE of 50 to compare to the AIF scans and at an optional NCE of 100.

An overview of the fragmentation behavior of pyoverdine PAO1, 206-12, 1-60, and 3G07 can be found in the electronic supplementary (Fig. S1-S2). For PAO1, a good fragmentation was achieved at an NCE of 35. Its Suc-Py consists of 8 amino acids of which the last four cyclize, forming a new amide bond. This seems to lead to a stabilizing effect. A similar observation was made for the Suc-Py of Py SA, also belonging to the class of cyclopeptides. Pyoverdines containing a C-terminal cyclo-hydroxyornithine such as Suc-Py from sample 1-60 started to fragment already at an NCE of 25. Even though the precursor was mostly intact, the highest diversity of fragments was present at this point. Using a higher collision energy produced solely more low $\mathrm{m} / \mathrm{z}$ fragments containing little to no structural information. Comparing the Suc-Py of 3G07 and 206-12, the difference in fragmentation of a cyclo-depsipeptide (presence of an ester bond) and a peptide ending with a free $\mathrm{COOH}$ group was observable. While the cyclo-depsipeptide was stable until 30 NCE, the uncyclized 206-12 pyoverdine fragmented at 25 NCE. This suggests again an increase of stability by cyclization.

Hence, multiple collision energies are necessary for a general applicable method. As cyclized pyoverdines tend to be more stable, the first structural information can be gained simply by determining the optimal collision energy. Recording additional MS/MS spectra at 50 and $100 \mathrm{NCE}$ allows to measure the pyoverdine characteristic fragment at $\mathrm{m} / \mathrm{z}, 204$ in large abundance.

As literature reports several cases of re-arrangement reactions during the fragmentation of multiply charged species [22], the $[M+\mathrm{H}]^{+}$ions were also chosen as a precursor, if present. In our case, all MS/MS spectra of the $[M+2 \mathrm{H}]^{2+}$ ions could be easily evaluated. Nevertheless, MS/MS parameters were set in such a way that our method can also cover the fragmentation of the $[M+\mathrm{H}]^{+}$ion during the same measurement run. Using a loop count of 12 , two coeluting precursor ions can be picked and fragmented at six different isolated NCEs $(20,25,30,35,50$, and 100) delivering at least 8 scans over the peak per collision energy. The resolution of 35,000, the long injection time, and AGC target improve the sensitivity compared to lower resolution settings. In this way, high-quality MS/MS spectra are obtained in a single run.

An elegant way to overcome various problems during the annotation of fragments is to record MS/MS spectra of at least two pyoverdine derivatives produced by a single bacterial strain instead of choosing only one precursor ion. The advantage of this procedure is illustrated in Fig. 8 where the fragmentation patterns of Glu-FerB, Suca-Py and Suc-Py of S3b09 are compared: As the peptide chain is identical for all pyoverdine derivatives, fragments that were broken off from the $\mathrm{C}$-terminus and that do not contain the chromophore together with its side chain (X, Y, and $\mathrm{Z}$ fragments) own the same $m / z$ signals in all fragmentation spectra (highlighted in green). In contrast to this, fragments including the chromophore/side chain unit (A, B, and C fragments) will result in different masses. This mass difference accounts $m / z-0.9840$ and $m / z+17.0629$ when comparing Suc-Py to Suca-Py and Glu-FerB, respectively (highlighted in red). As the side chain has little influence on the fragmentation behavior, similar fragment ion intensities are observed for all three compounds at the same collision energy. Thus, a comparison is easily possible.

\section{Assembling block by block: structural reconstruction}

The optimized fragmentation method was applied to all bacterial samples and the fragmentation interpretation pipeline was validated using the reference compounds of PAO1, 206-12, 1-60, and Py SA. Final elucidated peptide sequences are listed in Table 1 . An existing reference [14] was found for all but four peptide chains. Thus, 3A06, 3D19, S3g01, and 3B19 contain newly discovered pyoverdines with various $\mathrm{C}$-terminal endings according to the best of our knowledge. Their Suc-Py structures are shown in Fig. 9 (3-6). For each sample, a fragment list containing ppm deviations and a visualized chemical structure as well as the mainly used MS/MS spectra at a specific NCE of the corresponding Suc-Py are attached in the supplementary materials (Tables S3-S19).

In all cases, the Glu-FerB precursor was successfully identified and led to the determination of the Suc-Py and Suca-Py masses. For each sample, an ideal NCE value was found where sufficient fragments were present proving that the stepped collision values from 20 to 35 (a.u.) cover an ideal range. Thereby, linear pyoverdines tended to fragment at 20 and $25 \mathrm{NCE}$ while cyclized structures required a higher NCE of 30 or 35.

Adapting the nomenclature for peptide fragmentations, $\mathrm{B}$ and $\mathrm{Y}^{\prime \prime}$ ions are reported to be the most abundant ions in 
Fig. 8 a Visualization of $\mathrm{N}$ and C-terminal fragments for pyoverdine derivatives (variations of the side chain and/ or chromophore). b Exemplary MS/MS spectra of Suca-Py, Suc-Py, and Glu-FerB at NCE 25 found in the bacterial extract of S3b09 (green $=$ C-terminal fragments with identical $\mathrm{m} / \mathrm{z}$ value, red $=\mathrm{N}$-terminal fragments with a mass shift due to the side chain and/or chromophore variation) a

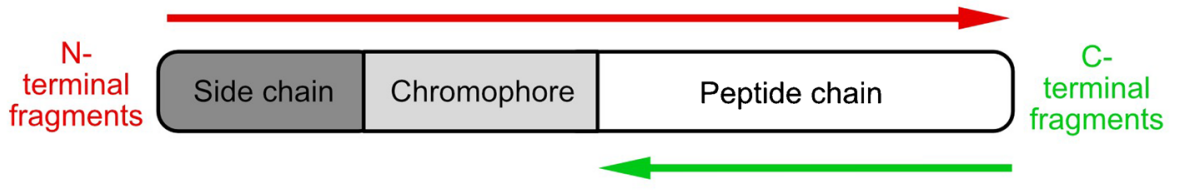

b

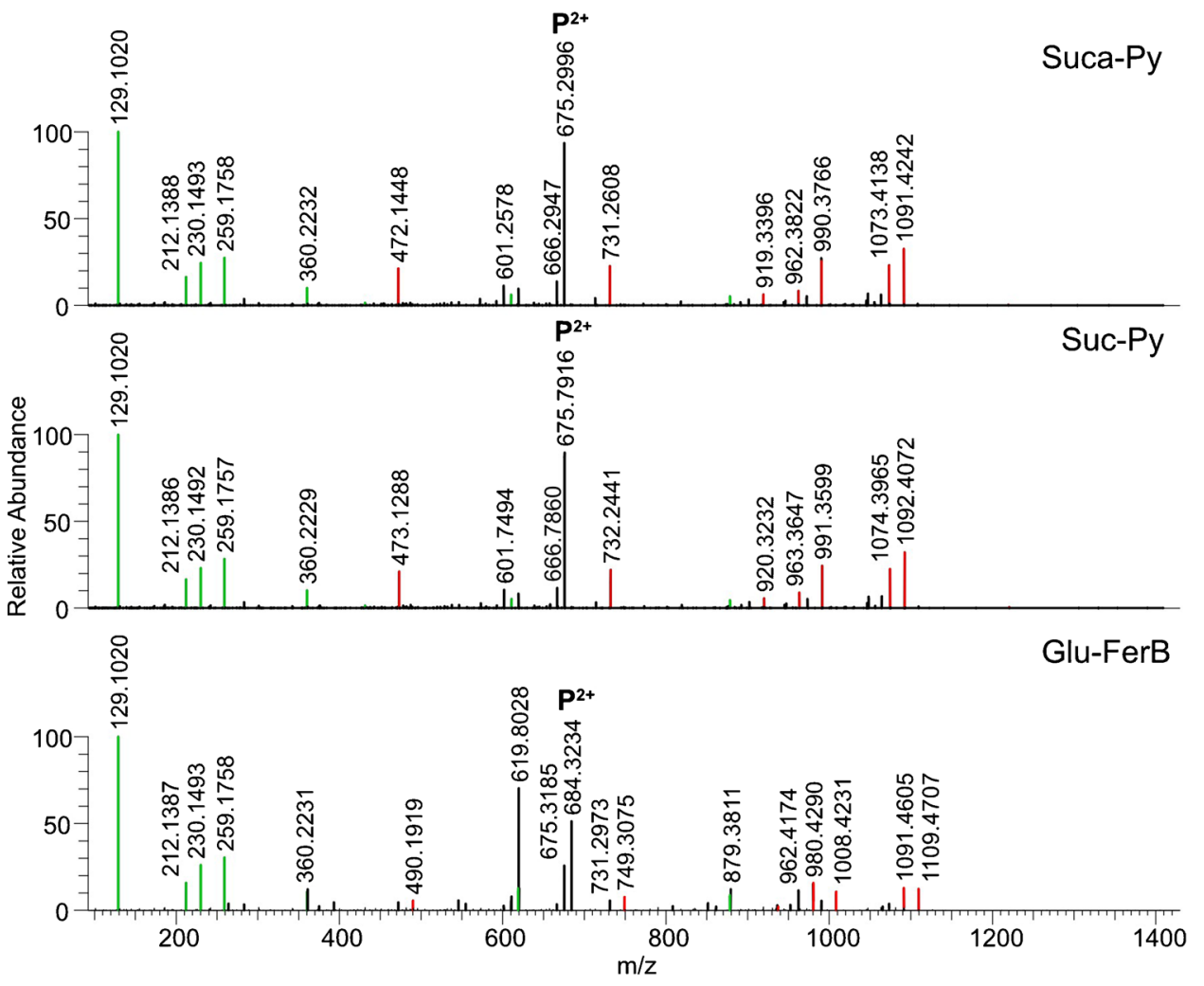

pyoverdine MS/MS fragmentation. As the $\mathrm{A}_{1}$ or $\mathrm{B}_{1}$ fragment are the easiest distinguishable signals, the reconstruction of the complete $\mathrm{p}$ eptide sequence is possible [15]. A short overview over the most expected $\mathrm{A}_{1}$ and $\mathrm{B}_{1}$ fragments based on the first amino acid in the peptide sequence is given in Table 2.

After the identification of the first A or B fragments, the pyoverdine peptide chain is subsequently elucidated by screening for the next fragment ions. Referring to the publication of Ye et al. [21], certain probabilities exist on the order of amino acids in a sequence. By using this information, the most likely occurring amino acids can be checked first. For lysine, occasionally a linkage of the $\varepsilon$ - instead of the $\alpha$-amino group to its preceding carboxyl group is observed. If an absence or very low intensity of the corresponding B fragment is noted, a valuable indication for an $\varepsilon$-linkage is given [29]. As all measurements were performed on a high-resolution mass spectrometer with a high mass precision, all measured masses including fragments should be compared to a calculated theoretical value as an additional support. As pyoverdines are natural products, only a limited set of amino acids are available to explain certain mass differences in the fragmentation spectra. Critical cases are Asn/Orn and Gln/Lys that possess the same nominal mass. However, their monoisotopic masses differ over 20 ppm. Hence, a tolerated error window of 5 ppm was chosen that is small enough to differentiate closely related amino acids but is also large enough to account for small intensity signals or potential matrix effects. Last but not least, the interpretation did not rely on the presence of characteristic fragments of certain amino acids, as these are reported to be sometimes missing [15].

The structure elucidation method was firstly applied to four pyoverdine references with already established structures. PAO1 and the Sigma Aldrich standard (Py SA) were chosen as both are cyclopeptides that are especially challenging to elucidate [30]. Additionally, pyoverdine 1-60 and 206-12 were employed ending in a C-terminal cyclohydroxyornithine and a free $\mathrm{COOH}$ group, respectively. Hence, structural varieties were covered that might show 


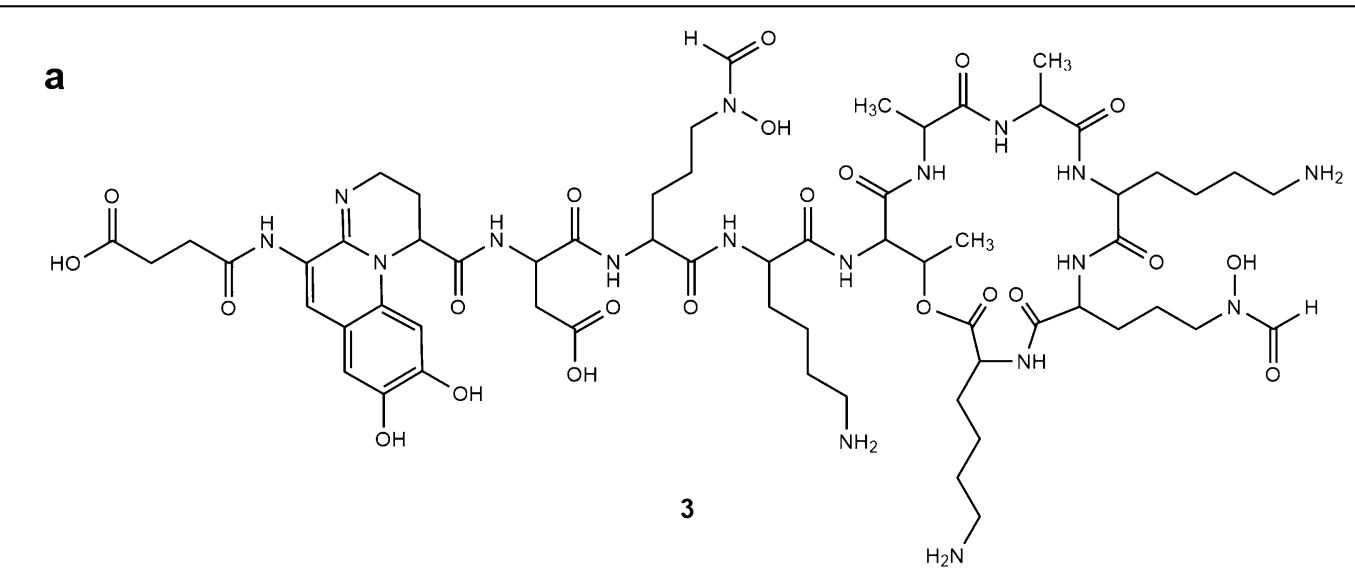<smiles>CC(O)C(NC(=O)C(CO)NC(=O)C(NC(=O)C(N)CCCCNC(=O)C(CC(=O)O)NC(=O)C1CCN=C2C(NC(=O)CCC(=O)O)=Cc3cc(O)c(O)cc3N21)C(O)C(=O)O)C(=O)NC(CO)C(=O)NC(CCCCN)C(=O)NC1CCCN(O)C1=O</smiles>

C<smiles>CC(=O)N(C)O</smiles><smiles>CCCC(NC(=O)C(C)NC(=O)C1CCN=C2C(NC(=O)CCC(=O)O)=Cc3cc(O)c(O)cc3N21)C(=O)NC(C)C(=O)NCC(=O)NC(CO)C(=O)NC(C)C(=O)NC(C(=O)NC(C(=O)O)C(C)O)C(O)C(=O)O</smiles><smiles>CC(NC(=O)C(CO)NC(=O)CNC(=O)CNC(=O)C(CCCO)NC(=O)C(C)NC(=O)C1CCN=C2C(NC(=O)CCC(=O)O)=Cc3cc(O)c(O)cc3N21)C(=O)NC(C(=O)NC(C(=O)O)C(C)O)C(O)C(=O)O</smiles>

Fig. 9 a Novel pyoverdine with a cyclo-depsipeptide chain found in sample 3B19 (3). b Novel pyoverdine ending with a cyclo-hydroxyornithine found in sample 3A06 (4). c Novel pyoverdines with a free ending C-terminal peptide chain found in sample S3g01 (5) and 3D19 (6)

different fragmentation behavior. The $[M+2 \mathrm{H}]^{2+}$ signal of the Suc-Py was used as the main source for structure elucidation as it was present in all samples.
An intense fragment at $m / z 417.1405\left(\mathrm{~A}_{1}\right)$ served as a starting point as serine is the first building block in all reference compounds followed by the continuous assembly of further A and B ions. Fragmentation of Suc-Py of PAO1 
Table 2 Expected MS/MS fragments depending on the side chain and the first amino acid residue as reported by Budzikiewicz et al. [15]

\begin{tabular}{lll}
\hline $\begin{array}{l}1^{\text {st }} \text { amino acid of the pyover- } \\
\text { dine peptide sequence }\end{array}$ & Succinic amide & Succinic acid \\
\hline Ser & $416.1565\left(\mathrm{~A}_{1}\right)$ & $417.1405\left(\mathrm{~A}_{1}\right)$ \\
Ala & $400.1615\left(\mathrm{~A}_{1}\right)$ & $401.1456\left(\mathrm{~A}_{1}\right)$ \\
$\varepsilon$-Lys & $457.2194\left(\mathrm{~A}_{1}\right)$ & $458.2034\left(\mathrm{~A}_{1}\right)$ \\
Lys & $485.2143\left(\mathrm{~B}_{1}\right)$ & $486.1983\left(\mathrm{~B}_{1}\right)$ \\
Asp & $472.1463\left(\mathrm{~B}_{1}\right)$ & $473.1303\left(\mathrm{~B}_{1}\right)$ \\
\hline
\end{tabular}

and Py SA resulted in more complex MS/MS spectra. Their peptide ring is opened during the fragmentation process in the HCD cell. Thereby, not only the $\varepsilon$-amide bond of the incorporated Lys group can be broken (that contributed to the ring closure) but the $\alpha$-amide bond, too. This results in $\mathrm{B}$ fragments that do not follow the usual order of the peptide sequence (fragment E, see Table S3-S4). For 206-12, initially, no $\mathrm{B}_{6}$ fragment was found leaving the two last amino acids unclear. However, a $\left[\mathrm{B}_{6}+\mathrm{H}\right]^{2+}$ was detected recognizable by the differing decimal places of the $\mathrm{m} / \mathrm{z}$ signal completing the structure. As Suc-Py 206-12 owns a Ser followed by a Dab group, a $[M+2 \mathrm{H}]^{2+}$ signal was recorded for a Dab condensed and a Dab uncondensed form. Both ions were separately chosen as a precursor for fragmentation and compared. A cleaner MS/MS spectrum was obtained for the uncondensed species making it the better choice for structure elucidation. MS/MS of Suc-Py from 1-60 gave a high-intensity signal at $\mathrm{m} / \mathrm{z} 131.0814$. This fragment belongs to a characteristic $\mathrm{Y}_{1}{ }_{1}$ ion that consists of terminal cyclohydroxyornithines. Taking this fragment into account, the effort to characterize an unfamiliar peptide can be reduced. In the end, all reference structures were correctly elucidated $a b$ inito, proving that the optimized fragmentation and interpretation procedure works/is valid.

All unknown samples could be confidently analyzed. The starting point of the fragment interpretation $\left(\mathrm{A}_{1}\right.$ or $\left.\mathrm{B}_{1}\right)$ was effortlessly detected in all cases. Similar to the reference compounds, Suc-Py from S3c13 and S3e20 started with a serine group and gave an intense signal at $\mathrm{m} / z, 417.1405$ $\left(\mathrm{A}_{1}\right)$. On the other hand, the Suc-Py produced by $3 \mathrm{~A} 06$, 3G07, 3B19, and S3b09 began with an aspartic acid $\left(\mathrm{B}_{1}\right.$ : $\mathrm{m} / \mathrm{z}$ 473.1303). Suc-Py starting with an alanine from sample 3D19, S3b16, and S3g01 resulted in a clear fragment at $m / z 401.1456\left(\mathrm{~A}_{1}\right)$. For the characterization of $\varepsilon$-Lys vs. Lys components, the lack of the corresponding $\mathrm{B}$ frag ment was the deciding factor for an $\varepsilon$-linkage as observed for 3A06, S3b09, and S3a20. In these cases, only a rather small signal for the A fragment was detected. For S3a05, 3B19, and al 1 remaining compounds, a clear $\mathrm{B}$ fragment incorporating a Lys was found proving the usual amino acid connectivity.
The pyoverdine of 3G07 and 3B19 turned out to be cyclodepsipeptides owning a ring system caused by a condensation of a threonine with the terminal lysine. Despite their cyclic structure, all A and B fragments necessary for their elucidation were easily located. Since the ester bond is readily hydrolyzed and broken, less fragments with an intact ring are observed. In contrast, amide bonds are more stable due to their double bond character that requires higher collision energies for their breakage. Thus, more complicated spectra are obtained when fragmenting cyclo-peptides.

Afterwards, the following A and/or B fragments could be determined. By comparison of at least two MS/MS spectra of different pyoverdine derivatives, the nature of fragment ions, C- or N-terminal, was readily pinpointed. One example of a completely annotate MS/MS spectrum is presented in Fig. 10 and Table 3 for the new compound 4 found in 3A06.

By using accurate high-resolution MS, the ppm error to theoretical values was finally calculated. In all our cases, this error was below $5 \mathrm{ppm}$.

To sum up, the structure of all pyoverdine samples was successfully elucidated, thanks to the well-specified methodology used in the measurement and interpretation of the MS/MS spectra. 3A06, 3D19, S3g01, and 3B19 owned new peptide sequences not reported previously resulting in a considerable extension of the currently known pyoverdine structure list [14].

\section{Mass calculator and fragment predictor for pyoverdines and derivatives}

While rapid MS-based protein sequencing is made possible by automated computational algorithms, pyoverdine structure elucidations were conducted mainly by hand until today. Whereas this might be feasible for few samples, a manual characterization is tedious and takes many hours for multiple compounds. Furthermore, pyoverdine masses are typically reported from the corresponding Suc-Py molecule without decimal numbers, which is insufficient for high-resolution mass spectrometry. Also, no sum formulas are usually indicated and only the peptide chain sequence is stated [14]. Hence, a pyoverdine mass calc ulator using easily editable drop-down menus was developed in Excel (the spreadsheet is available in the supplementary materials). Exact masses of atoms were taken from IUPAC [31]. Based on the publications of Budzikiewicz et al. [15], the following aspects were taken into account: the variability of the side chain, the chromophore, the peptide sequence, and the peptide end. All to date published partial structures were considered and made available for selection. Thus, the monoisotopic mass of all pyoverdine derivates can be predicted together with their $[M+\mathrm{H}]^{+}$and $[M+2 \mathrm{H}]^{2+} \mathrm{m} / z$ values.

Furthermore, a mass difference table was created to faster scrutinize the full scan for possible pyoverdines and other 
Fig. $10 \mathrm{MS} / \mathrm{MS}$ spectrum of the Suc-Py $[M+2 \mathrm{H}]^{2+}$ ions $(\mathrm{m} / \mathrm{z}$ $633.26821)$ of $3 \mathrm{~A} 06(\mathrm{NCE}=25)$

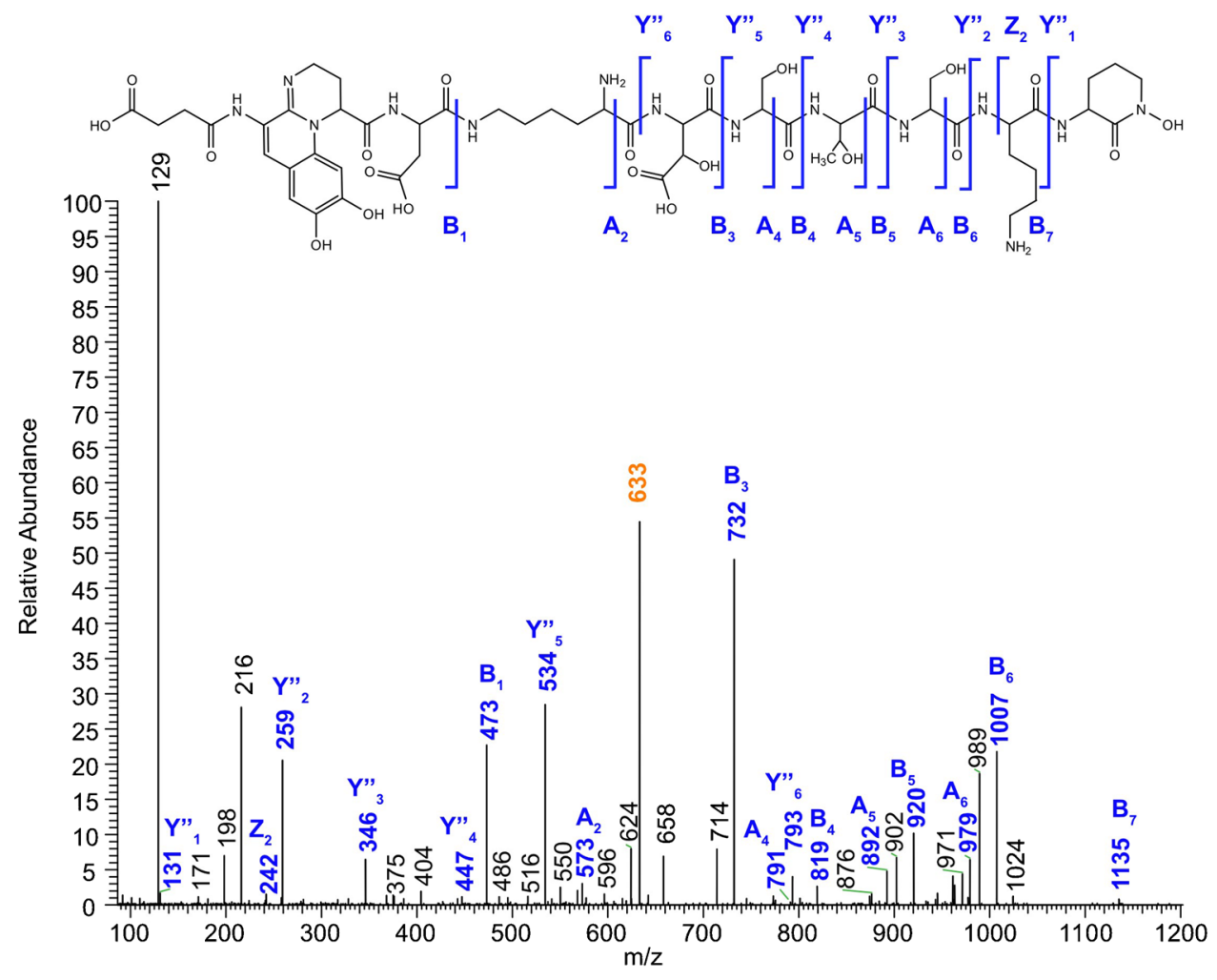

Table 3 Identified fragments in the MS/MS spectrum of the Suc-Py $[M+2 \mathrm{H}]^{2+}$ ions $(\mathrm{m} / \mathrm{z}$ 633.26821) found in extract 3 A06 at NCE 25. Ppm deviations are calculated based on the difference between theoretical and measured masses. *Theoretical masses were calculated using the mass and fragment predictor for pyoverdines and its derivatives

\begin{tabular}{|c|c|c|c|c|}
\hline & Formula & Theoretical mass* $(\mathrm{m} / \mathrm{z})$ & Measured mass $(\mathrm{m} / \mathrm{z})$ & Deviation (ppm) \\
\hline$[M+2 \mathrm{H}]^{2+}$ & $\mathrm{C}_{52} \mathrm{H}_{78} \mathrm{~N}_{14} \mathrm{O}_{23}$ & 633.26821 & 633.26699 & -1.93 \\
\hline $\mathrm{A}_{2}$ & $\mathrm{C}_{26} \mathrm{H}_{33} \mathrm{~N}_{6} \mathrm{O}_{9}$ & 573.23035 & 573.23188 & 2.66 \\
\hline $\mathrm{A}_{4}$ & $\mathrm{C}_{33} \mathrm{H}_{43} \mathrm{~N}_{8} \mathrm{O}_{15}$ & 791.28424 & 791.28388 & -0.45 \\
\hline $\mathrm{A}_{5}$ & $\mathrm{C}_{37} \mathrm{H}_{50} \mathrm{~N}_{9} \mathrm{O}_{17}$ & 892.33192 & 892.33267 & 0.84 \\
\hline $\mathrm{A}_{6}$ & $\mathrm{C}_{40} \mathrm{H}_{55} \mathrm{~N}_{10} \mathrm{O}_{19}$ & 979.36395 & 979.36458 & 0.65 \\
\hline $\mathrm{B}_{1}$ & $\mathrm{C}_{21} \mathrm{H}_{21} \mathrm{~N}_{4} \mathrm{O}_{9}$ & 473.13030 & 473.13006 & -0.52 \\
\hline $\mathrm{B}_{3}$ & $\mathrm{C}_{31} \mathrm{H}_{38} \mathrm{~N}_{7} \mathrm{O}_{14}$ & 732.24713 & 732.24671 & -0.57 \\
\hline $\mathrm{B}_{4}$ & $\mathrm{C}_{34} \mathrm{H}_{43} \mathrm{~N}_{8} \mathrm{O}_{16}$ & 819.27915 & 819.27946 & 0.37 \\
\hline $\mathrm{B}_{5}$ & $\mathrm{C}_{38} \mathrm{H}_{50} \mathrm{~N}_{9} \mathrm{O}_{18}$ & 920.32683 & 920.32572 & -1.21 \\
\hline $\mathrm{B}_{6}$ & $\mathrm{C}_{41} \mathrm{H}_{55} \mathrm{~N}_{10} \mathrm{O}_{20}$ & 1007.35886 & 1007.35886 & -0.00 \\
\hline $\mathrm{B}_{7}$ & $\mathrm{C}_{47} \mathrm{H}_{67} \mathrm{~N}_{12} \mathrm{O}_{21}$ & 1135.45382 & 1135.45068 & -2.77 \\
\hline $\mathrm{Y}^{\prime \prime}{ }_{1}$ & $\mathrm{C}_{5} \mathrm{H}_{11} \mathrm{~N}_{2} \mathrm{O}_{2}$ & 131.08150 & 131.08171 & 1.57 \\
\hline $\mathrm{Y}^{\prime \prime}{ }_{2}$ & $\mathrm{C}_{11} \mathrm{H}_{23} \mathrm{~N}_{4} \mathrm{O}_{3}$ & 259.17647 & 259.17652 & 0.20 \\
\hline$Y^{\prime \prime}{ }_{3}$ & $\mathrm{C}_{14} \mathrm{H}_{28} \mathrm{~N}_{5} \mathrm{O}_{5}$ & 346.20850 & 346.20850 & 0.00 \\
\hline $\mathrm{Y}^{\prime \prime}{ }_{4}$ & $\mathrm{C}_{18} \mathrm{H}_{35} \mathrm{~N}_{6} \mathrm{O}_{7}$ & 447.25617 & 447.25742 & 2.79 \\
\hline $\mathrm{Y}^{\prime}{ }_{5}$ & $\mathrm{C}_{21} \mathrm{H}_{40} \mathrm{~N}_{7} \mathrm{O}_{9}$ & 534.28820 & 534.28826 & 0.11 \\
\hline$Y^{\prime \prime}{ }_{7}$ & $\mathrm{C}_{31} \mathrm{H}_{57} \mathrm{~N}_{10} \mathrm{O}_{14}$ & 793.40502 & 793.40428 & -0.94 \\
\hline $\mathrm{Z}_{2}$ & $\mathrm{C}_{11} \mathrm{H}_{20} \mathrm{~N}_{3} \mathrm{O}_{3}$ & 242.14992 & 242.14994 & 0.09 \\
\hline
\end{tabular}

derivatives. If one has the accurate mass for the ferribactin precursor, the Excel file can predict quickly the $[M+2 \mathrm{H}]^{2+}$ $\mathrm{m} / \mathrm{z}$ values of all other theoretically possible combinations of side chain and chromophore. Thus, additional time can be saved when looking for the masses of Suc-Py or Suca-Py.
Last but not least, a fragmentation predictor for A, B, C, $\mathrm{X}, \mathrm{Y}, \mathrm{Y}^{\prime \prime}$, and $\mathrm{Z}$ ions was created. This is of massive advantage when interpreting fragmentation spectra as decimal numbers can be immediately compared from predicted to measured values such as presented in Table 3 . Even though it is designed for linear peptides, it partially works for cyclized 
compounds, too, especially when taking a water loss from the cyclization reaction into account. Electrons were minded for accurate masses.

All in all, the pyoverdine calculator produces not only accurate monoisotopic masses but also supports fragmentation interpretation. The calculator and fragmentation predictor were tested and verified with the known pyoverdine structures and proved to be working smoothly.

\section{Conclusion}

Siderophores gained a large interest in research due to their pathogen control properties making systematic structure elucidation methods at high-throughput desirable. With this study, a generalized workflow was established using pyoverdine of fluorescent Pseudomonas spp. as a model siderophore. This workflow was validated using 17 different pyoverdine extracts. Sample preparation takes place by a small-scale SPE saving time and resources to obtain pure solutions for analysis. The herein reported UHPLC conditions deliver an excellent separation in only $15 \mathrm{~min}$ using reserved phase column chemistry. The process to identify pyoverdines was simplified by the search for the ferribactin fragment in an AIF scan and is of great advantage when analyzing unusual Pseudomonas species that produce atypical pyoverdine derivatives such as isopyoverdine. Our targeted MS/MS experiments using multiple collision energies can be applied to a broad range of pyoverdines showing various fragmentation behavior resulting in high-quality MS/MS spectra. Finally, the lack of a mass calculator and fragmentation predictor for pyoverdine and its derivatives was addressed. Here, we make a simple but efficient Excel tool available that speeds up the work-intensive comparison of measured and theoretical masses that is necessary when working with high-resolution mass spectrometry. This analytical approach can serve as a basis for the development of high-throughput methods to characterize other siderophore classes and secondary metabolites from small sample volumes, thereby accelerating research progress.

Supplementary Information The online version contains supplementary material available at https://doi.org/10.1007/s00216-022-03907-w.

Funding Open access funding provided by University of Zurich. This study was funded in part by the Swiss National Science Foundation SNSF (grant no. 31003A_182499 to RK).

Availability of data and material Fully described methods are provided using commercially available materials. Origin of bacterial cultures is fully disclosed. For the MS/MS fragmentation, exact measured masses as well as the raw MS/MS spectra are given.
Code availability Not applicable.

\section{Declarations}

Conflict of interest The authors declare no competing interests.

Open Access This article is licensed under a Creative Commons Attribution 4.0 International License, which permits use, sharing, adaptation, distribution and reproduction in any medium or format, as long as you give appropriate credit to the original author(s) and the source, provide a link to the Creative Commons licence, and indicate if changes were made. The images or other third party material in this article are included in the article's Creative Commons licence, unless indicated otherwise in a credit line to the material. If material is not included in the article's Creative Commons licence and your intended use is not permitted by statutory regulation or exceeds the permitted use, you will need to obtain permission directly from the copyright holder. To view a copy of this licence, visit http://creativecommons.org/licenses/by/4.0/.

\section{References}

1. Demain AL, Fang A. The natural functions of secondary metabolites. Adv Biochem Eng Biotechnol. 2000;69:1-39. https://doi. org/10.1007/3-540-44964-7_1.

2. Waters CM, Bassler BL. Quorum sensing: cell-to-cell communication in bacteria. Annu Rev Cell Dev Biol. 2005;21:319-46. https://doi.org/10.1146/annurev.cellbio.21.012704.131001.

3. Braud A, Hoegy F, Jezequel K, Lebeau T, Schalk IJ. New insights into the metal specificity of the Pseudomonas aeruginosa pyoverdine-iron uptake pathway. Environ Microbiol. 2009;11:1079-91. https://doi.org/10.1111/j.1462-2920.2008.01838.x.

4. Rosenberg E, Ron EZ. High- and low-molecular-mass microbial surfactants. Appl Microbiol Biotechnol. 1999;52:154-62. https:// doi.org/10.1007/s002530051502.

5. Cezard C, Farvacques N, Sonnet P. Chemistry and biology of pyoverdines, pseudomonas primary siderophores. Curr Med Chem. 2015;22:165-86. https://doi.org/10.2174/09298673216661410111 94624.

6. Demain AL. Pharmaceutically active secondary metabolites of microorganisms. Appl Microbiol Biotechnol. 1999;52:455-63. https://doi.org/10.1007/s002530051546.

7. Demain AL, Sanchez S. Microbial drug discovery: 80 Years of progress. J Antibiot (Tokyo). 2009;62:5-16. https://doi.org/10. 1038/ja.2008.16.

8. Marchant R, Banat IM. Microbial biosurfactants: challenges and opportunities for future exploitation. Trends Biotechnol. 2012;30:558-65. https://doi.org/10.1016/j.tibtech.2012.07.003.

9. Saha M, Sarkar S, Sarkar B, Sharma BK, Bhattacharjee S, Tribedi P. Microbial siderophores and their potential applications: a review. Environ Sci Pollut Res. 2016;23:3984-99. https://doi.org/ 10.1007/s11356-015-4294-0.

10. Hider RC, Kong X. Chemistry and biology of siderophores. Nat Prod Rep. 2010;27:637-57. https://doi.org/10.1039/b906679a.

11. Gu S, Wei Z, Shao Z, Friman VP, Cao K, Yang T, Kramer J, Wang X, Li M, Mei X, Xu Y, Shen Q, Kümmerli R, Jousset A. Competition for iron drives phytopathogen control by natural rhizosphere microbiomes. Nat Microbiol. 2020;5:1002-10. https://doi.org/10. 1038/s41564-020-0719-8.

12. Figueiredo ART, Özkaya Ö, Kümmerli R, Kramer J. Siderophores drive invasion dynamics in bacterial communities through their dual role as public good versus public bad. Ecol Lett. 2022;25:138-50. https://doi.org/10.1111/ele.13912. 
13. Demange P, Wendenbaum S, Linget C, Mertz C, Cung MT, Dell A, Abdallah MA. Bacterial siderophores : structure and NMR assignment of pyoverdins $\mathrm{Pa}$, siderophores of Pseudomonas aeruginosa ATCC 15692. Biol Met. 1990;3:155-70. https://doi.org/ 10.1007/BF01140574.

14. Meyer J-M, Gruffaz C, Raharinosy V, Bezverbnaya I, Schäfer M, Budzikiewicz H. Siderotyping of fluorescent Pseudomonas: molecular mass determination by mass spectrometry as a powerful pyoverdine siderotyping method. Biometals. 2008;21:259-71. https://doi.org/10.1007/s10534-007-9115-6.

15. Budzikiewicz H, Schafer M, Meyer J-M. Siderotyping of fluorescent pseudomonads - problems in the determination of molecular masses by mass spectrometry. Mini Rev Org Chem. 2007;4:24653. https://doi.org/10.2174/157019307781369968.

16. Budzikiewicz H, Schäfer M, Fernández DU, Matthijs S, Cornelis P. Characterization of the chromophores of pyoverdins and related siderophores by electrospray tandem mass spectrometry. Biometals. 2007;20:135-44. https://doi.org/10.1007/s10534-006-9021-3.

17. Ringel MT, Brüser T. The biosynthesis of pyoverdines. Microb. Cell. 2018;5:424-37. https://doi.org/10.15698/mic2018.10.649.

18. Baune M, Qi Y, Scholz K, Volmer DA, Hayen H. Structural characterization of pyoverdines produced by Pseudomonas putida KT2440 and Pseudomonas taiwanensis VLB120. Biometals. 2017;30:589-97. https://doi.org/10.1007/s10534-017-0029-7.

19. Wei H, Aristilde L. Structural characterization of multiple pyoverdines secreted by two Pseudomonas strains using liquid chromatography-high resolution tandem mass spectrometry with varying dissociation energies. Anal Bioanal Chem. 2015;407:4629-38. https://doi.org/10.1007/s00216-015-8659-5.

20. Sarkar PK, Prajapati PK, Shukla VJ, Ravishankar B, Choudhary AK. Characterization of microbial siderophores by mass spectrometry. Mass Spectrom Rev. 2016;35:35-47. https://doi.org/10. $1002 / \mathrm{mas}$.

21. Ye L, Ballet S, Hildebrand F, Laus G, Guillemyn K, Raes J, Matthijs S, Martins J, Cornelis P. A combinatorial approach to the structure elucidation of a pyoverdine siderophore produced by a Pseudomonas putida isolate and the use of pyoverdine as a taxonomic marker for typing P. putida subspecies. Biometals. 2013;26:561-75. https://doi.org/10.1007/s10534-013-9653-z.

22. Fuchs R, Budzikiewicz H. Rearrangement reactions in the electrospray ionization mass spectra of pyoverdins+. Int J Mass Spectrom. 2001;210(211):603-12. https://doi.org/10.1016/S13873806(01)00408-0.
23. Fuchs R, Schäfer M, Geoffroy V, Meyer J-M. Siderotyping a powerful tool for the characterization of pyoverdines. Curr Top Med Chem. 2001;1:31-57. https://doi.org/10.2174/1568026013 395542.

24. Hohlneicher U, Hartmann R, Taraz K, Budzikiewicz H. Pyoverdin, ferribactin, azotobactin - a new triade of siderophores from pseudomonas chlororaphis ATCC 9446 and its relation to pseudomonas fluorescens ATCC 13525. Zeitschrift für Naturforsch - Sect C J Biosci. 1995;50:337-44. https://doi.org/10.1515/ znc-1995-5-602.

25. Smith EE, Sims EH, Spencer DH, Kaul R, Olson MV. Evidence for diversifying selection at the pyoverdine locus of Pseudomonas aeruginosa. J Bacteriol. 2005;187:2138-47. https://doi.org/10. 1128/JB.187.6.2138-2147.2005.

26. Visca P, Imperi F, Lamont IL. Pyoverdine siderophores: from biogenesis to biosignificance. Trends Microbiol. 2007;15:22-30. https://doi.org/10.1016/j.tim.2006.11.004.

27. Butaite E, Baumgartner M, Wyder S, Kümmerli R. Siderophore cheating and cheating resistance shape competition for iron in soil and freshwater Pseudomonas communities. Nat Commun. 2017;8:414. https://doi.org/10.1038/s41467-017-00509-4.

28. Marchia I, Vietteb V, Badoudac F, Fathib M, Saugyc M, Rudaza S, Veutheya J-L. Characterization and classification of matrix effects in biological samples analyses. J Chromatogr A. 2010;1217:40718. https://doi.org/10.1016/j.chroma.2009.08.061.

29. Budzikiewicz H. Siderophores of the Pseudomonadaceae sensu stricto (fluorescent and non-fluorescent Pseudomonas spp.). Prog Chem Org Nat Prod. 2004;87:81-237. https://doi.org/10.1007/ 978-3-7091-0581-8_2.

30. Schäfer M, Fuchs R, Budzikiewicz H, Springer A, Meyer J-M, Linscheid M. Structure elucidation of cyclic pyoverdins and examination of rearrangement reactions in MS/MS experiments by determination of exact product ion masses. J mass Spectrom. 2007;41:1162-70. https://doi.org/10.1002/jms.

31. De Laeter JR, Böhlke JK, De Bièvre P, Hidaka H, Peiser HS, Rosman KJR, Taylor PDP. Atomic weights of the elements: Review 2000 (IUPAC Technical Report). Pure Appl Chem. 2003;75:683800. https://doi.org/10.1351/pac200375060683.

Publisher's note Springer Nature remains neutral with regard to jurisdictional claims in published maps and institutional affiliations. 